OPEN ACCESS

Edited by: Santosh Kumar,

Liaocheng University, China

Reviewed by:

Tony Jose,

Karunya Institute of Technology and

Sciences, India

Madan M. Upadhyay,

Indian Institute of Technology

Dhanbad, India

*Correspondence:

Sushank Chaudhary sushankchaudhary@gmail.com

Lunchakorn Wuttisittikulkij

Lunchakorn.W@chula.ac.th

Specialty section:

This article was submitted to

Optics and Photonics,

a section of the journal

Frontiers in Physics

Received: 10 August 2021

Accepted: 23 August 2021

Published: 07 September 2021

Citation:

Chaudhary S, Wuttisittikulkij L,

Nebhen J, Tang $X$, Saadi M,

Al Otaibi S, Althobaiti A, Sharma A and Choudhary S (2021) Hybrid MDM-

PDM Based Ro-FSO System for

Broadband Services by Incorporating

Donut Modes Under Diverse

Weather Conditions.

Front. Phys. 9:756232.

doi: 10.3389/fphy.2021.756232

\title{
Hybrid MDM-PDM Based Ro-FSO System for Broadband Services by Incorporating Donut Modes Under Diverse Weather Conditions
}

\section{Sushank Chaudhary ${ }^{1 *}$, Lunchakorn Wuttisittikulkij ${ }^{1 *}$, Jamel Nebhen ${ }^{2}$, Xuan Tang $^{3}$, Muhammad Saadi ${ }^{4}$, Sattam Al Otaibi ${ }^{5}$, Ahmed Althobaiti ${ }^{5}$, Abhishek Sharma ${ }^{6}$ and Sunita Choudhary ${ }^{1}$}

${ }^{1}$ Wireless Communication Ecosystem Research Unit, Department of Electrical Engineering, Chulalongkorn University, Bangkok, Thailand, ${ }^{2}$ College of Computer Engineering and Sciences, Prince Sattam Bin Abdulaziz University, Alkharj, Saudi Arabia, ${ }^{3}$ Quanzhou Institute of Equipment Manufacturing, Chinese Academy of Sciences, Quanzhou, China, ${ }^{4}$ Department of Electrical Engineering, University of Central Punjab, Lahore, Pakistan, ${ }^{5}$ Department of Electrical Engineering, College of Engineering, Taif University, Taif, Saudi Arabia, ${ }^{6}$ Department of Electronics and Communication Engineering, Guru Nanak Dev University, Amritsar, India

High-speed data demand in sensitive locations has prompted new wireless technologies to grow in areas like hospitals for bio-sensor data transmission between doctors and patients. However, interference of electromagnetic spectrum or highly sensitive medical equipment in such locations can prevent radio waves which can further compromise the health of patients. Radio over Free Space Optics (Ro-FSO) can fulfil high-speed data demand in such locations without any such interference. However, the Ro-FSO performance is highly influenced by different adverse weather conditions, particularly haze and rainfall, which further cause attenuation in the transmission path of Ro-FSO systems. These atmospheric turbulences mainly affect the transmission link range of RoFSO systems. In this work, Ro-FSO system is designed by incorporating hybrid mode division multiplexing (MDM) and polarization division multiplexing (PDM) schemes to deliver four independent channels, each carrying $10 \mathrm{Gbps}$ data upconverted to $40 \mathrm{GHz}$ radio signal, over $3.4 \mathrm{~km}$ free space optical link operating under clear weather conditions. In addition to this, the proposed Ro-FSO link is subjected to different weather conditions, particularly partially hazy/rainy and dense fog/very rainy. The reported results indicate the achievement of acceptable bit error rate $\left(B E R \approx 10^{-3}\right)$ for all channels up to $3400 \mathrm{~m}$ FSO link under clear weather conditions, $1000 \mathrm{~m}$ under partially haze/rain and $620 \mathrm{~m}$ under dense fog/heavy rain.

Keywords: radio over free space optics, mode division multiplexing, polarization division multiplex, partially hazy, dense fog

\section{INTRODUCTION}

The current pandemic situation of COVID-19 has not only affected global healthcare in general but also raised questions on the effectiveness of epidemic response mechanisms across the world. In terms of screening the infected individuals and frontline healthcare personnel, communication and data exchange systems have a crucial role to play. Particularly in high-density countries like Thailand, 


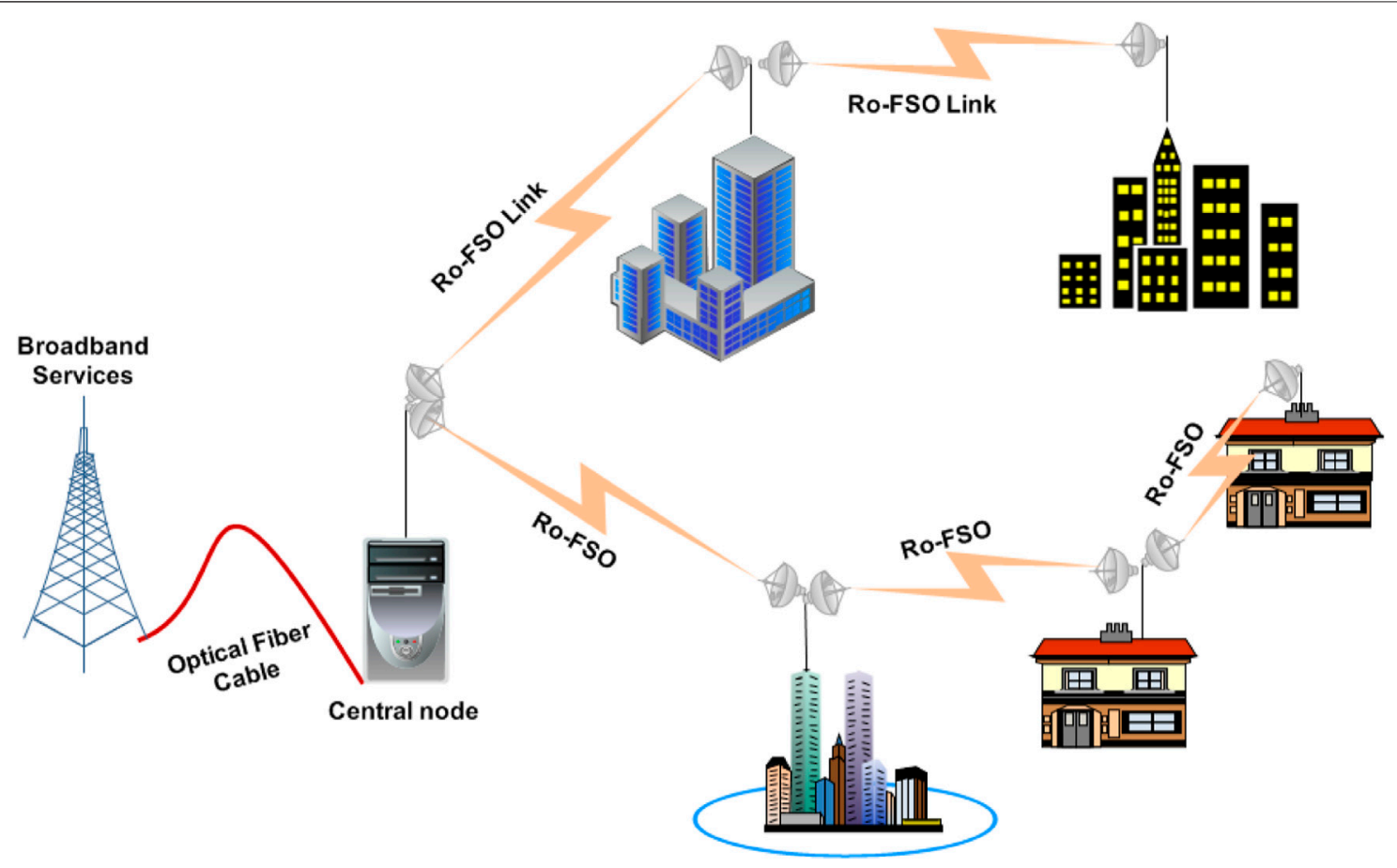

FIGURE 1 | Ro-FSO topology for broadband service.

China, and India, it is essential to update such systems for controlling the ongoing pandemic outbreak. Telecommunication operators can install high-speed broadband networks in hospitals by using innovative applications to drive emergency response mechanisms digitally and accurately. Both high and low frequencies can be used by broadband networks to provide highest speeds on millimeter waves with frequencies between 30 and $300 \mathrm{GHz}$. However, these radio millimeter waves are not permitted in sensitive medical locations since it can interfere with crucial medical equipment which can impact patients' health. Therefore, such sensitive locations are always considered as challenging locations to connect with broadband services. Moreover, growing population has caused substantial increase in data demand which further compels researchers to explore new-generation communication technologies. According to a survey by Ericsson, annual mobile data traffic saw an increase of $65 \%$ in 2015 which is assumed to grow ten times bigger by the end of 2021 [1]. The issue of bandwidth increase of the existing wireless radio networks can be resolved by decreasing the cell size to accommodate more users. Spectral congestions can be mitigated by operating frequency bands in microwave or millimeter bands [2]. Because of this, the service area requires multiple base stations resulting in its complexed structure and higher cost. Free Space Optics (FSO) has thrived in application in high-speed wireless networks due to its features for smooth traffic functioning in optical fibers [3-5]. Moreover, another FSO-based study [6] used point-to-point laser signals for negligible interception to secure transmission. Studies [7-9] have also reported features of FSO including its high capacity, less power consumption, light weight, smaller size and cost-effective implementation charges. Radio over Free Space Optical (Ro-FSO) communication can be an excellent solution to fulfill the demands of high-speed data by combining radio technology with optical wireless technology at lower cost [10, 11]. As modern cities are growing bigger and bigger, digging of lands to install optical fiber cables is not feasible. Thus, Ro-FSO can be a potential carrier to deliver various high-speed digital services such as high-speed internet, video on demand, and Triple Play Services (TPS) without using optical fiber cables $[12,13]$ as shown in Figure 1. Ro-FSO can handle the increasing mobile subscribers by transmitting RF signals through high-speed optical carrier that does not cost much or need any licensing.

Some of the major features of Ro-FSO include no license requirement, low power consumption and ease of deployment; however, its performance is limited in atmospheric turbulences such as fog, rain, scintillations, and fog $[4,14,15]$. Atmospheric turbulences cause temporal random fluctuations of the refractive index through the optical channel due to temperature, wind variations, and pressure [16]. This fluctuation in refractive index further results in phase shift of optical signals transmitting through the atmosphere leading to attenuation in the wave front. To improve the performance, many researchers have proposed orthogonal frequency division multiplexing (OFDM) scheme by compromising the complexity and cost of FSO systems. In 2016 [17], authors have transmitted $1.6 \mathrm{Gbps}$ data over $1.8 \mathrm{~km}$ FSO link by employing OFDM scheme under heavy rainfall conditions. In 2017 [18], authors have used 16 QAM-OFDM encoding scheme to transmit $20 \mathrm{Gbps}$ data over $10 \mathrm{~km}$ single mode fiber link and $300 \mathrm{~mm}$ FSO link. In 

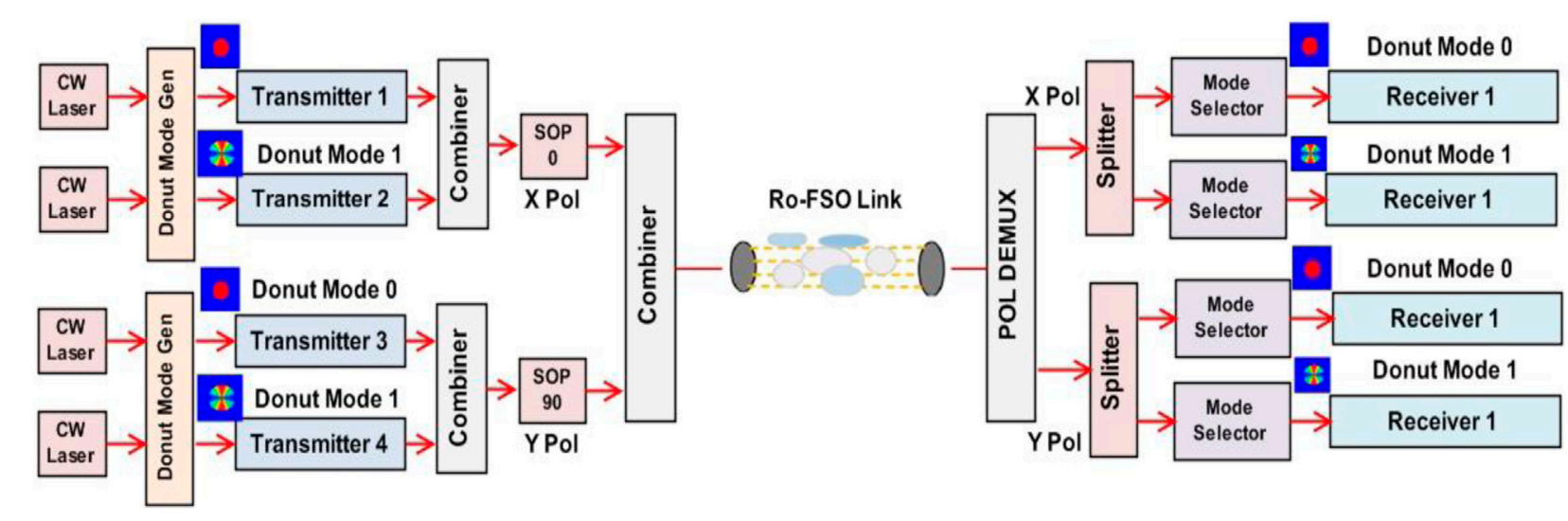

FIGURE 2 | MDM-PDM-Ro-FSO transmission system.

2018 [19], authors have proposed OFDM-based FSO link by incorporating non line-of-sight assisted relay. Recently in 2019 [20], authors have proposed hybrid polarization division multiplexing (PDM) and OFDM schemes to transmit data over $5 \mathrm{~km}$ FSO link. OFDM can improve the FSO system performance by providing high spectral bandwidth, firmness against multipath path fading effects and narrowband interferences. However, this increases the cost and complexity of FSO transmitter and receiver. On-off key encoding schemes can be an attractive solution to reduce the cost of and simplify FSO communication system. But it also needs to compromise the transmission capacity and distance. Whereas mode division multiplexing (MDM) becomes an attractive solution to increase the transmission capacity of multimode transmission systems by simultaneously transmitting different channels on different modes. The last few years have witnessed remarkable adoption of MDM scheme in FSO systems. It uses eigen modes instead of wavelength which can enhance the capacity of Ro-FSO. Eigen modes can be generated by using mechanisms such as Photonic Crystal Fibers (PCF) or Spatial Light Modulators (SLM). The spectral bandwidth of Ro-FSO can further be increased by incorporating PDM [21] with MDM.

\section{MODELING OF MDM-PDM-Ro-FSO SYSTEM.}

Figure 2 shows how the hybrid MDM-PDM scheme is designed for transmitting four independent channels by using OptiSystem ${ }^{\mathrm{TM}}$ software. Each channel can support the data rate of $10 \mathrm{Gbps}$ upconverted to $40 \mathrm{GHz} \mathrm{mm}$ carrier. A continuous wavelength (CW) laser with the wavelength of $1,552.5 \mathrm{~nm}$ is used to excite the particular donut mode by using donut mode generator. The power of laser is set at $0 \mathrm{dBm}$. Transmitter 1 is operated on donut mode 0 and Transmitter 2 is operated on donut mode 1 . The output of these transmitters is integrated. The state of polarization controller with azimuth of $0^{0}$ is used for maintaining the polarization state (X Polarization). Similarly, transmitters 3 and 4 are operated on donut modes 0 and 1 , respectively. The output of transmitters 3 and 4 is

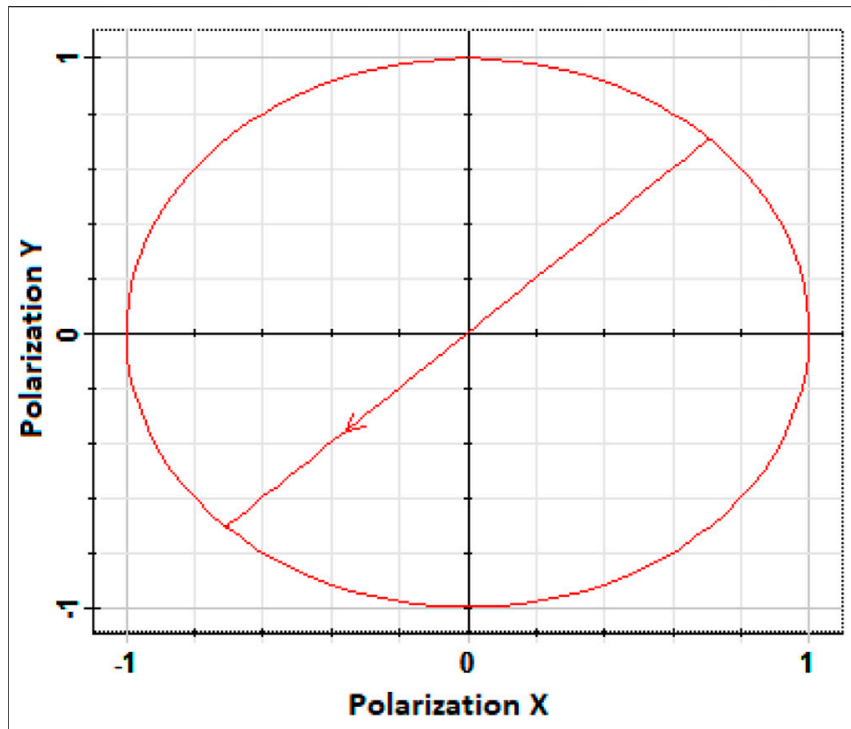

FIGURE 3 | Maintained polarization states.

combined and a polarization state (Y Polarization) is maintained by providing azimuth of $90^{\circ}$ through the state of polarization controller. The output of the states of polarization controllers is combined and transmitted through free space link. The output of link is amplified by using optical amplifier with the gain of $18 \mathrm{~dB}$. Figure 3 shows the maintained polarization states for $\mathrm{X}$ and $\mathrm{Y}$ polarizations. $\mathrm{X}$ and $\mathrm{Y}$ polarizations are separated by using polarization splitter at the receiver side. The polarization states are further split into two parts corresponding to two modes transmitted at the transmitter side. Mode selector is used to select the particular mode required for the receiver side.

Figure 4 shows the schematic diagram of transmitter and receiver in the proposed MDM-PDM-Ro-FSO system. $10 \mathrm{Gbps}$ data are encoded by employing low-cost NRZ encoding scheme and then upconverted to $40 \mathrm{GHz} \mathrm{mm}$ wave by using mixer as shown in Figure 5. Sine wave generator is used to generate the $40 \mathrm{GHz} \mathrm{mm}$ signal. 


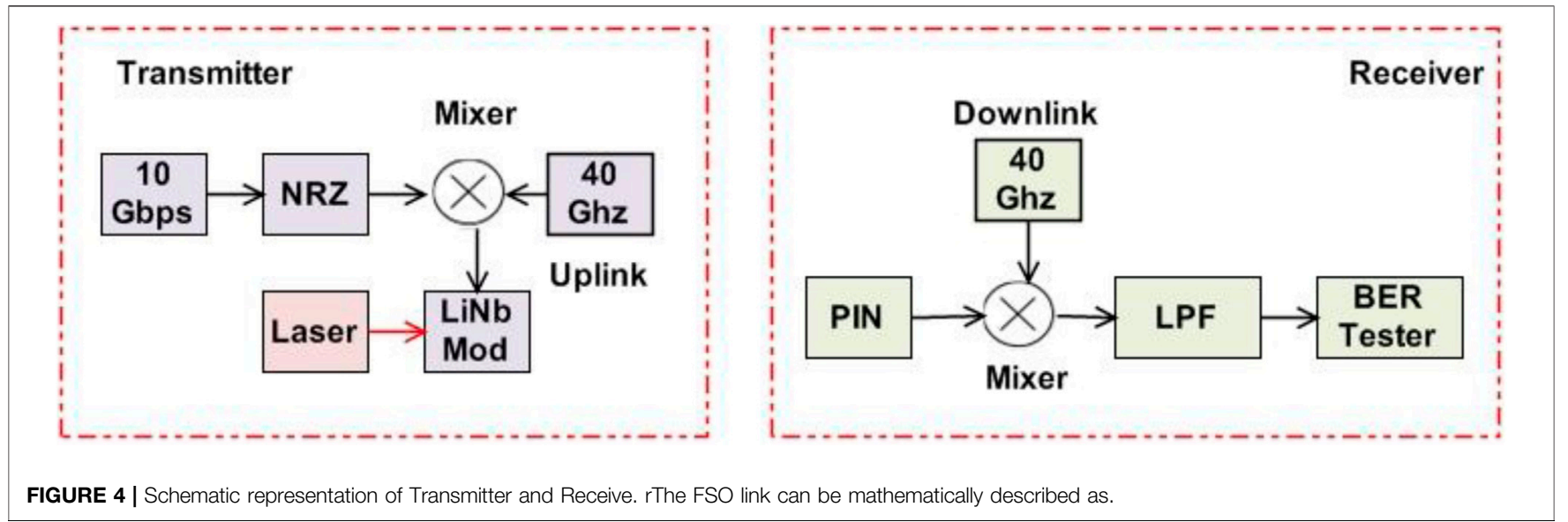

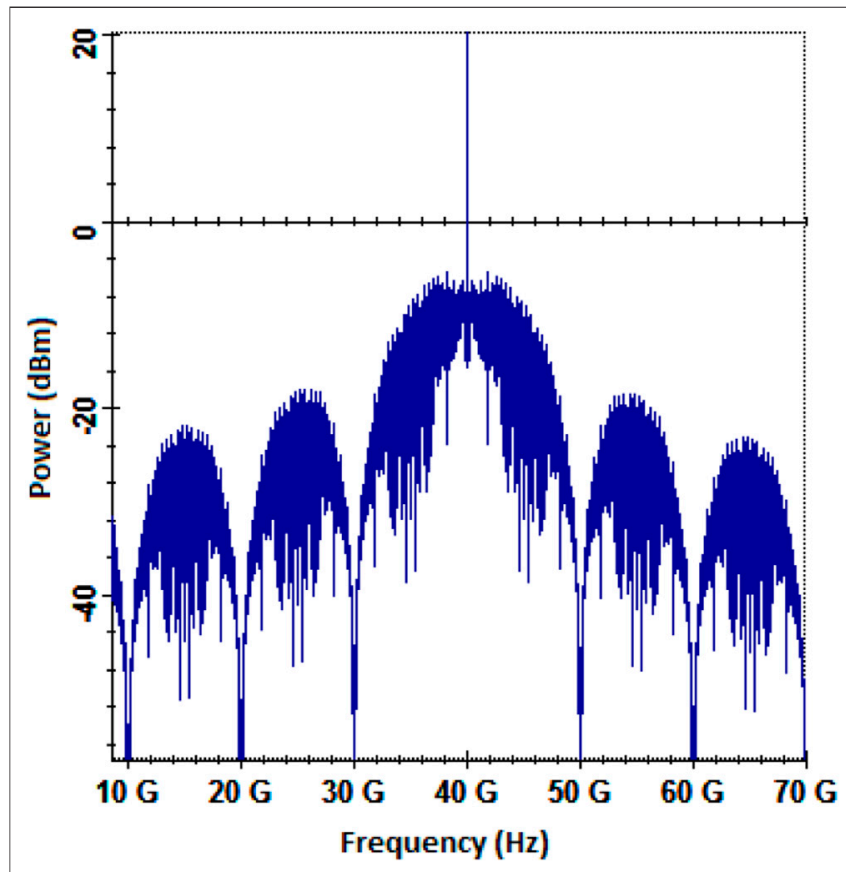

FIGURE 5 | Measured Upconverted $40 \mathrm{GHz}$ radio carrier.

Lithium Niobate optical modulator is used for modulating the $40 \mathrm{GHz}$ upconverted wave through optical carrier. The receiver consists of PIN photodiode which further converts the optical signal back to the corresponding electrical signal. The output of photodetector is then mixed with $40 \mathrm{GHz}$ radio signal to attain the process of down conversion.

After the down conversion process, low-pass filter is used to recover the original baseband signal.

$$
P_{\text {Received }}=P_{\text {Transmitted }} \frac{d_{R}^{2}}{\left(d_{T}+\vartheta R\right)^{2}} 10^{-\alpha \frac{R}{10}}
$$

In this FSO link equation, $P_{\text {Received }}$ refers to the received power at the receiving end of telescope and $P_{\text {Transmitted }}$ the transmitted
TABLE 1 | Attenuation value under different atmospheric turbulences.

\begin{tabular}{lc} 
Clear sky & $\mathbf{0 . 4 6 8}$ \\
\hline Partially Hazy/Rainy & 12 \\
Dense Fog/Nery Hazy & 25
\end{tabular}

TABLE 2 | MDM-PDM-Ro-FSO modeling parameters.

\begin{tabular}{lll} 
Component name & \multicolumn{1}{c}{ Parameter name } & Value \\
\hline Spatial Laser & Wavelength & $850 \mathrm{~nm}$ \\
& Power & $0 \mathrm{dBm}$ \\
& Mode outer radius & $5 \mathrm{\mu m}$ \\
& Linewidth & $10 \mathrm{MHz}$ \\
& Noise dynamic & $3 \mathrm{~dB}$ \\
MZM Modulator & Extinction Ratio & $30 \mathrm{~dB}$ \\
FSO & Transmitter Aperture Diameter & $5 \mathrm{~cm}$ \\
& Receiver Aperture Diameter & $20 \mathrm{~cm}$ \\
APD & Beam Divergence & $2 \mu \mathrm{rad}$ \\
& Gain & 3 \\
& Responsivity & $1 \mathrm{AW}$ \\
& lonization ratio & 0.9
\end{tabular}

power at the transmitting end of telescope. $d_{\mathrm{T}}$ is the transmitter's and $d_{\mathrm{R}}$ the receiver's aperture diameters. $\vartheta$ is defined as beam divergence and FSO range is denoted by $R$ and attenuation range is denoted by $\alpha$. Beer-Lambert law is used for calculating the attenuation phenomena which can be represented as follows [22]:

$$
H(\lambda, L)=\exp (\lambda, L)
$$

In this equation, " $\lambda$ " refers to wavelength, " $L$ " the propagation distance and " $\mathrm{H}$ " the loss coefficient. Another model on FSO attenuation that depends upon visibility factor known as Kim's attenuation model can be represented mathematically as [23]:

$$
a(d B / K m)=\frac{3.91}{v}\left(\frac{\lambda}{550}\right)^{-q}
$$

where $v$ refers to meteorological visibility, $\lambda$ operating wavelength and $q$ a factor depending on the size distribution of atmospheric particles. 

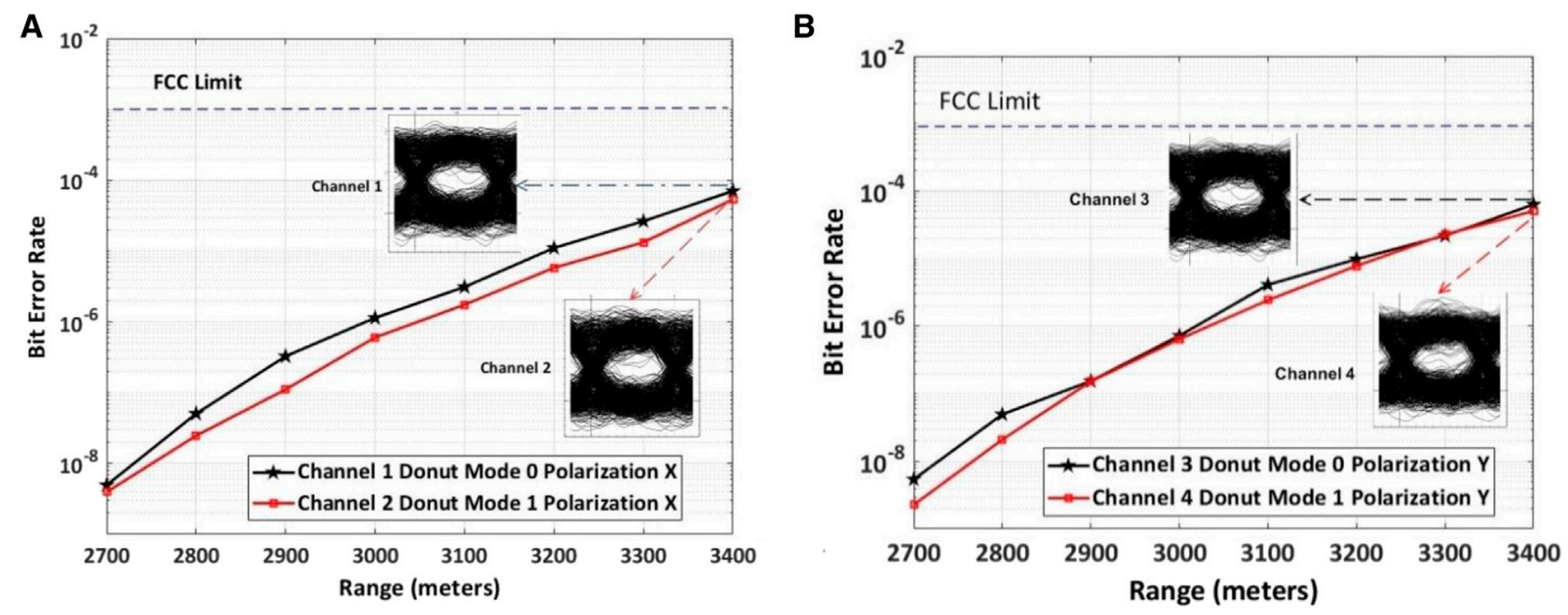

FIGURE 6 | Computed BER (clear weather conditions) (A) Channel 1 and Channel 2 (X Polarization) (B) Channel 3 and Channel 4 (Y Polarization).
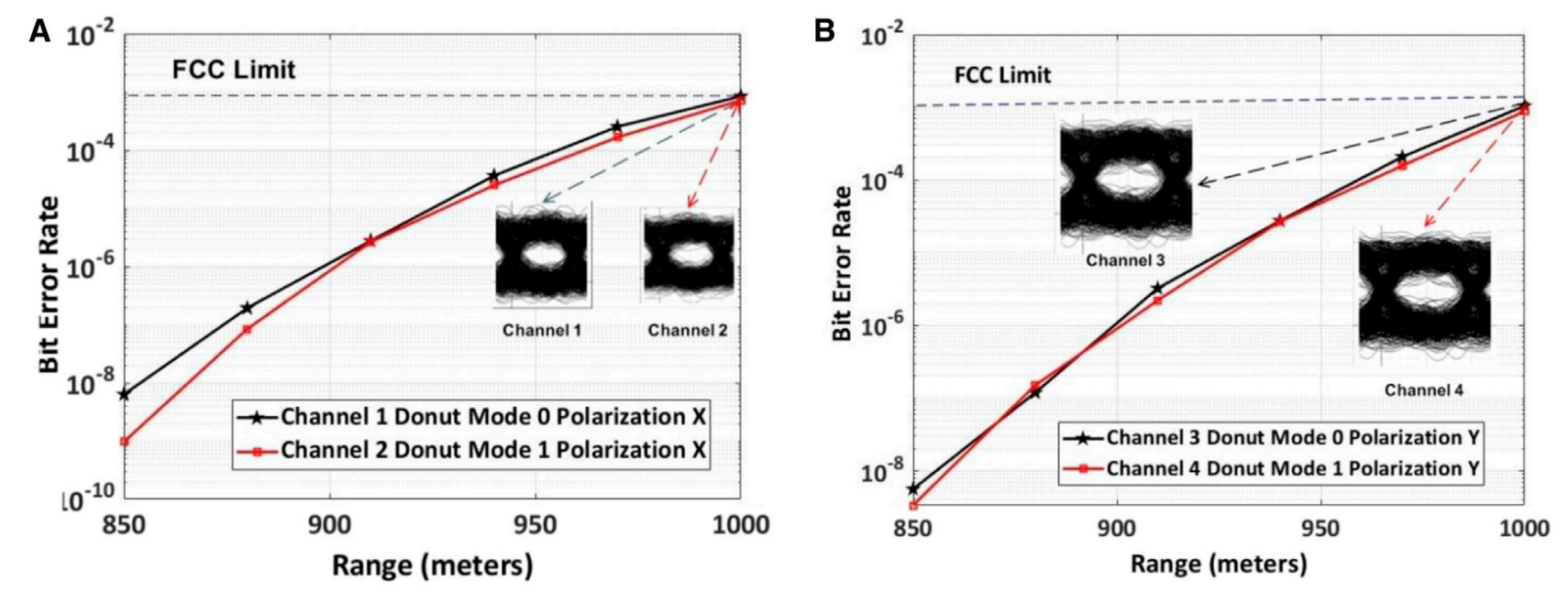

FIGURE 7 | Measured BER under partially hazy/rainy atmospheric condition (A) Channel 1 and Channel 2 (B) Channel 3 and Channel 4

Under the impact of different rainfall, the following equation can calculate the attenuation [24]:

$$
\alpha(d B / k m)=1.076 \times R^{0.67}
$$

where $R$ is the amount of rainfall. Table 1 shows the different values of atmospheric turbulences [25] and Table 2 shows the parameters to model the proposed MDM-PDM-RoFSO link.

\section{MODELING OBSERVATIONS AND DISCUSSIONS}

The results obtained from the modeling of the proposed MDM-PDMRo-FSO system are presented and discussed in this section. Figure 6 depicts the measured BERs for channels 1 and 2 operated on donut modes 0 and 1 , respectively with $\mathrm{X}$ polarization as well as for channels 3 and 4 with Y polarization state with respect to Ro-FSO link distance under clear weather conditions. As shown in Figure 6A, the computed BER values for Channel 1 and Channel 2 are $10^{-9}, 10^{-7}$ and $10^{-5}$ at the FSO transmission link of 2,700, 3,200, and 3,400 m. Similarly, for Channel 3 and Channel 4 , the BER values are computed as $10^{-9}, 10^{-6}$ and $10^{-5}$ at the FSO transmission link of $2,700 \mathrm{~m}, 3,200 \mathrm{~m}$ and $3,400 \mathrm{~m}$ as shown in Figure 6B.

Therefore, under clear weather conditions, all channels achieved the BER of $10^{-4}$ which is below the range of acceptable $\mathrm{BER} \approx 10^{-3}$ (FCC limit) at the FSO link of $3400 \mathrm{~m}$ with required opening of eye diagrams. However, Channel 2 and Channel 4 operated on donut mode 1 are slightly affected more as compared to Channel 1 and Channel 3 operated on donut mode 0 . In another case, the Ro-FSO link is subjected to partially hazy/rainy atmospheric conditions and computed BER is shown in Figure 7. The values of BER for Channel 1 

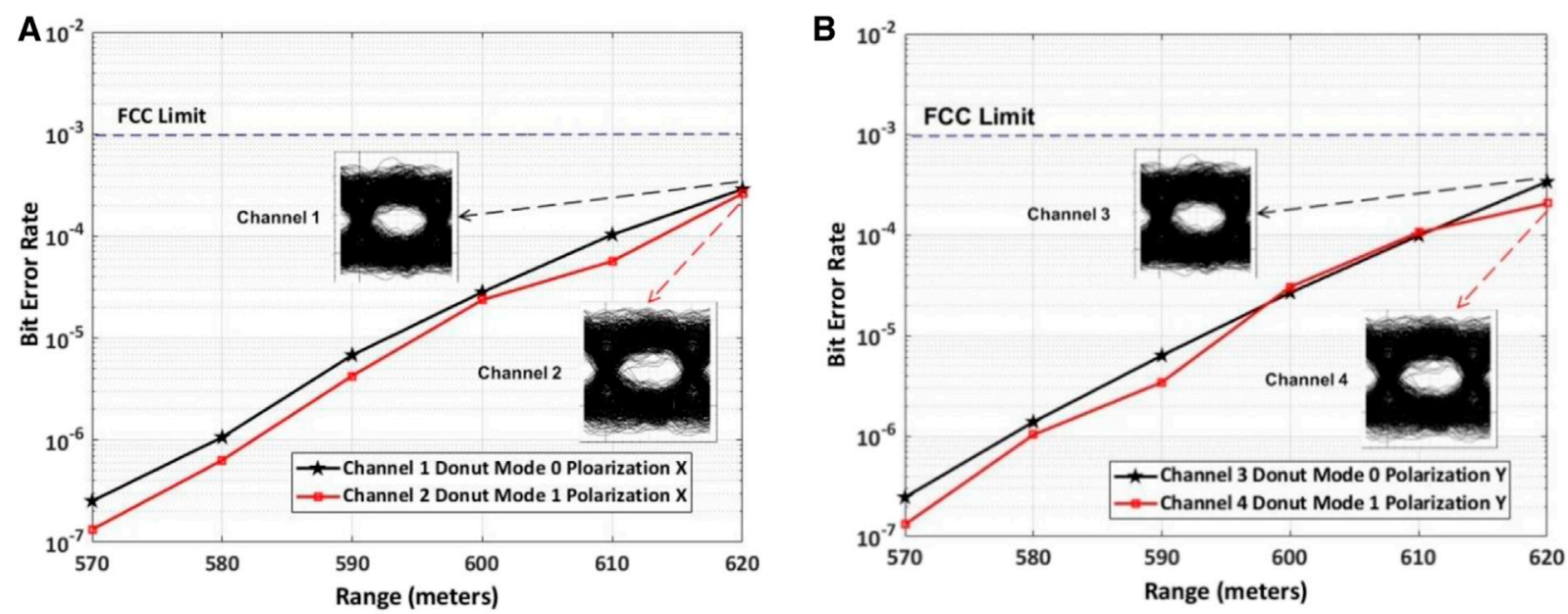

FIGURE 8 | Computed BER (dense fog/very hazy atmospheric conditions) (A) Channel 1 and Channel 2 (X Polarization) (B) Channel 3 and Channel 4 (Y Polarization).

are computed as $10^{-7}, 10^{-5}$ and $10^{-3}$ at the FSO transmission link of 850,940 and $1000 \mathrm{~m}$. Similarly for Channel 2, it is computed as $10^{-9}$, $10^{-5}$ and $10^{-3}$ at the FSO transmission link of 850,940 and $1000 \mathrm{~m}$. As shown in Figure 7B, the values of computed BER for Channel 3 and Channel 4 are $10^{-9}, 10^{-5}$ and $10^{-3}$ at the FSO transmission link of 850, 940 and $1000 \mathrm{~m}$.

Figure 8 shows the measured BER for all channels under the influence of dense fog/very hazy atmospheric conditions. For Channel 1 and Channel 2, the values of BER are computed as $10^{-7}, 10^{-5}$, and $10^{-4}$ at the FSO transmission link of 570, 600 and $620 \mathrm{~m}$.

Similarly as shown in Figure 8B, for Channel 3 and Channel 4, the values of BER are computed as $10^{-7}, 10^{-5}$ and $10^{-4}$ at the FSO transmission link of 570, 600 and $620 \mathrm{~m}$.

Thus, when the proposed Ro-FSO link is operated in clear weather conditions, the computed BER shows transmission of all the channels up to $3400 \mathrm{~m}$ FSO link with acceptable BER $\approx 10^{-3}$ and eye diagrams. After the FSO transmission distance, BER value increases beyond the FCC limit. Moreover, when the proposed Ro-FSO link is operated in partial haze/rain, the FSO transmission distance reduces to $1000 \mathrm{~m}$ with acceptable BER and eye diagrams. Furthermore, when the proposed Ro-FSO link is operated in dense fog/heavy rain, the FSO transmission distance reduces to $620 \mathrm{~m}$ with acceptable BER and eye diagrams.

\section{CONCLUSION}

In this work, Ro-FSO link is designed for transmission of broadband services by employing hybrid MDM and PDM schemes. Donut modes 0 and 1 were used for MDM scheme whereas NRZ scheme was used for encoding the pseudorandom data. The designed MDM-PDM-Ro-FSO link could transmit four independent channels each with $10 \mathrm{Gbps}$ data upconverted to $40 \mathrm{GHz}$ radio signal. Under the clear weather condition, the proposed MDM-PDM-Ro-FSO link can transmit $40 \mathrm{Gbps}$ data up to $3400 \mathrm{~m}$ with acceptable $\mathrm{BER} \approx 10^{-3}$. However, when the weather condition changed to partial haze/rain, the proposed MDM-PDM-Ro-FSO link could withstand only up to $1000 \mathrm{~m}$ due to attenuation. Furthermore, when the atmospheric condition changed from partial haze/rain to dense fog/heavy rain, the proposed link could withstand up to $650 \mathrm{~m}$ only with acceptable BER.

\section{DATA AVAILABILITY STATEMENT}

The raw data supporting the conclusion of this article will be made available by the authors, without undue reservation.

\section{AUTHOR CONTRIBUTIONS}

SC: Conceptualization, methodology, investigation, writing-original draft. LW: supervision, resources, project administration, funding acquisition. JN and XT: supervision, funding acquisition. MS , SA, and AA: Methodology, Funding Acquisition, resources. AS and SC: data curation and visualization.

\section{FUNDING}

This research project is supported by the Second Century Fund (C2F), Chulalongkorn University, Thailand. This research work is also funded by TSRI Fund (CU_FRB640001_01_21_8).

\section{ACKNOWLEDGMENTS}

The authors also would like to thank Taif University Researchers supporting project number (TURSP-2020/228), Taif University, Taif, Saudi Arabia. 


\section{REFERENCES}

1. de la Fuente A, Leal RP, Armada AG. New Technologies and Trends for Next Generation Mobile Broadcasting Services. IEEE Commun Mag (2016) 54: 217-23. doi:10.1109/mcom.2016.1600216rp

2. Zin A, Bongsu M, Idrus S, Zulkifli N. An Overview of Radio-Over-Fiber Network Technology. In International Conference On Photonics (2010). p. 1-3. doi:10.1109/icp.2010.5604429

3. Henniger $\mathrm{H}$, Wilfert $\mathrm{O}$. An Introduction to Free-Space Optical Communications. Radioengineering (2010) 19:203-12.

4. Malik A, Singh P. Free Space Optics: Current Applications and Future Challenges. Int J Opt (2015) 2015. doi:10.1155/2015/945483

5. Lim C, Wang K, Nirmalathas A. Optical Wireless Communications for HighSpeed In-Building Personal Area Networks. In Transparent Optical Networks (ICTON). Kuching, Sarawak, Malaysia.: International Conference on (20162016). p. 1-4. doi:10.1109/icton.2016.7550662

6. Majumdar AK. Fundamentals of Free-Space Optical (FSO) Communication System. In Advanced Free Space Optics (FSO). Springer (2015). p. 1-20. doi:10.1007/978-1-4939-0918-6_1

7. Siegel T, Chen S-P. Investigations of Free Space Optical Communications under Real-World Atmospheric Conditions. Wireless Pers Commun (2021) 116:475-90. doi:10.1007/s11277-020-07724-1

8. Sarangal H, Singh A, Malhotra J, Chaudhary S. A Cost Effective $100 \mathrm{Gbps}$ Hybrid MDM-OCDMA-FSO Transmission System under Atmospheric Turbulences. Opt Quant Electron (2017) 49:184. doi:10.1007/s11082-0171019-2

9. Anandkumar D, Sangeetha R. A Survey on Performance Enhancement in Free Space Optical Communication System through Channel Models and Modulation Techniques. Opt Quan Elect (2021) 53:1-39. doi:10.1007/ s11082-020-02629-6

10. Amphawan A, Chaudhary S, Neo T-K, Kakavand M, Dabbagh M. Radio-overfree Space Optical Space Division Multiplexing System Using 3-core Photonic crystal Fiber Mode Group Multiplexers. Wireless Netw (2021) 27:211-25. doi:10.1007/s11276-020-02447-4

11. Kuppusamy PG, Rajkumar K, Maheswar R, Rani SS, Amiri IS. A Long-Reach Radio over Free Space Optics (Ro-FSO) System Using Hybrid Orthogonal Frequency Division Multiplexing (OFDM)-multibeam Concept with Enhanced Detection. J Opt Commun 1:2020.

12. Mandal GC, Mukherjee R, Das B, Patra AS. Next-generation Bidirectional Triple-Play Services Using RSOA Based WDM Radio on Free-Space Optics PON. Opt Commun (2018) 411:138-42. doi:10.1016/ j.optcom.2017.11.033

13. Chaudhary S, Choudhary S, Tang X, Wei X. Empirical Evaluation of HighSpeed Cost-Effective Ro-FSO System by Incorporating OCDMA-PDM Scheme under the Presence of Fog. J Opt Commun (2020) 1. doi:10.1515/ joc-2019-0277

14. Chaudhary S, Amphawan A. The Role and Challenges of Free-Space Optical Systems. J Opt Commun (2014) 35:327-34. doi:10.1515/joc-2014-0004

15. Chaudhary S, Lin B, Tang X, Wei X, Zhou Z, Lin C, et al. 40 Gbps-80 GHz PSKMDM Based Ro-FSO Transmission System. Opt Quant Electron (2018) 50:321. doi:10.1007/s11082-018-1592-z
16. Xiaoming Zhu X, Kahn JM. Free-space Optical Communication through Atmospheric Turbulence Channels. IEEE Trans Commun (2002) 50: 1293-300. doi:10.1109/tcomm.2002.800829

17. Rashidi F, He J, Chen L. Performance Investigation of FSO-OFDM Communication Systems under the Heavy Rain Weather. J Opt Commun (2017) 39:37-42. doi:10.1515/joc-2016-0085

18. Perez J, Chicharro F, Ortega B, Mora J, "On the Evaluation of an Optical OFDM Radio over FSO System with IM-DD for High-Speed Indoor Communications," in 2017 19th International Conference on Transparent Optical Networks (ICTON), 2017, pp. 1-4.doi:10.1109/icton.2017.8025112

19. Kumar P, Thakor S. Performance of OFDM-FSO Link with Analog Network Coding. Photon Netw Commun (2018) 35:210-24. doi:10.1007/s11107-0170730-z

20. Kaur G, Srivastava D, Singh P, Parasher Y. Development of a Novel Hybrid PDM/OFDM Technique for FSO System and its Performance Analysis. Opt Laser Tech (2019) 109:256-62. doi:10.1016/j.optlastec.2018.08.008

21. Sumathi K, Balasaraswathi M, Boopathi C, Singh M, Malhotra J, Dhasarathan V. Design of 3.84 Tbps Hybrid WDM-PDM Based Inter-satellite Optical Wireless Communication (IsOWC) System Using Spectral Efficient Orthogonal Modulation Scheme. J Ambient Intelligence Humanized Comput (2020) 1-9.

22. Gebhart M, Leitgeb E, Muhammad SS, Flecker B, Chlestil C, Al Naboulsi M, et al. Measurement of Light Attenuation in Dense Fog Conditions for FSO Applications. In Atmospheric Optical Modeling. Measurement: and Simulation (2005). p. 58910K. doi:10.1117/12.614830

23. Shakir F, Ali MAA, Ameer F. Utilization of MIMO Concept for Optical Communication System under Fog Condition. ECTI-EEC (2019) 17:130-5. doi:10.37936/ecti-eec.2019172.219183

24. Kumar Giri R, Patnaik B. Bit Error Rate Performance Analysis of Hybrid Subcarrier Intensity Modulation-Based FSO with Spatial Diversity in Various Weather Conditions. J Opt Commun (2019) 40:307-14. doi:10.1515/joc-20170073

25. Israr A, Israr A, Khan F, Khan F. Optimal Modulation Technique for MIMO FSO Link. Wireless Personal Commun (2019) 1-20. doi:10.1007/s11277-019-06586-6

Conflict of Interest: The authors declare that the research was conducted in the absence of any commercial or financial relationships that could be construed as a potential conflict of interest.

Publisher's Note: All claims expressed in this article are solely those of the authors and do not necessarily represent those of their affiliated organizations, or those of the publisher, the editors and the reviewers. Any product that may be evaluated in this article, or claim that may be made by its manufacturer, is not guaranteed or endorsed by the publisher.

Copyright (c) 2021 Chaudhary, Wuttisittikulkij, Nebhen, Tang, Saadi, Al Otaibi, Althobaiti, Sharma and Choudhary. This is an open-access article distributed under the terms of the Creative Commons Attribution License (CC BY). The use, distribution or reproduction in other forums is permitted, provided the original author(s) and the copyright owner(s) are credited and that the original publication in this journal is cited, in accordance with accepted academic practice. No use, distribution or reproduction is permitted which does not comply with these terms. 